\title{
Numerical Simulation on Two Droplets Burning at Different Droplet Radius Ratios
}

\author{
Zifeng Hong \\ NO.703 Research Institute of CSIC \\ Harbin, China
}

Jing Zhao *

NO.703 Research Institute of CSIC

Harbin, China

E-mail: zhaojingcopaee@163.com

* Corresponding Author

\author{
Xiaobin Zhang \\ Institute of Refrigeration and Cryogenics, \\ Zhejiang University \\ Hangzhou, China
}

\author{
Longbin Yang \\ College of Power and Energy Engineering, \\ Harbin Engineering University \\ Harbin, China \\ Yanjun Li \\ College of Power and Energy Engineering, \\ Harbin Engineering University \\ Harbin, China
}

\begin{abstract}
It has some significance to study the influences of the droplet radius ratios on spray combustion due to the varying droplet radius ratios among droplets in the actual spray combustion. The volume of fluid (VOF) formulation is applied to model the combustion process of two decane droplets with different radiuses in a hot convective flow and the effects of the droplet ratios on burning rates of two droplets at fixed droplet center spacing are analyzed. The results show: when the burning rates of the front and the back droplets compare with that of the corresponding single droplet, the interaction reduces the burning rate of either droplet under different droplet radius ratios. For the same initial droplet radius, the burning rate of droplet located at the front is larger than that at the back under different droplet radius ratios. The results obtained provide some reference for the basic researches of spray combustion.
\end{abstract}

Keywords-Two Droplets; Burning Rate; Droplet Radius Ratio; Volume of Fluid Two-Phase Flow; Evaporation and Burning

\section{INTRODUCTION}

The study of droplets and sprays has developed rapidly over the past two decades because of their many important applications, from automobile engine combustion to drug aerosols $^{[1]}$. In the actual spray combustion, the droplet radius ratios among droplets are not the same, and always change. Therefore, it has some significance to study about the influences of the droplet radius ratios on spray combustion. In resent years, the studies ${ }^{[2-5]}$ about the influences of the droplet initial diameter on the single droplet burning are increasing. However, the researches about the effects of droplet radius ratios on spray combustion considering the droplets interaction are not plenty. In the static environment, the influences of the droplet radius ratios on the evaporation and combustion of the droplets were studied by [6,7]. In the air flow environment, the effects of the droplet radius ratios $(0.85$ and 1.5) on the burning rules of the droplets were studied by [8]. The burning characteristics of methanol porous with different radius ratios (2 and 0.5 ) were studied by experiment and numerical simulation ${ }^{[9]}$. Therefore, the research on the effects of droplet radius ratios on burning characteristics of two droplets enriches the basic researches of spray combustion. The results obtained provide some reference for the basic researches of spray combustion.

In this paper, the influences of droplet radius ratios on the burning rates of two droplets at fixed droplet center spacing are studied by using the VOF two-phase flow model. The computational domain adopts the axisymmetric model and the droplet deformation, the variable properties of gas phase and liquid phase, heat and mass transfer between gas-liquid two-phase are considered. Irreversible one-step reaction is used.

\section{PHYSICAL MODEL}

Figure 1 shows the physical configuration of two different $n$-decane droplets burning at fixed droplet center spacing. The whole computation domain is $20 \mathrm{~mm}$ long and $5 \mathrm{~mm}$ wide along the flow direction, which is believed to be large enough to include the influencing region of two droplets burning ${ }^{10]}$. The air flow has pressure $P=0.1 \mathrm{MPa}$, temperature $T=1100 \mathrm{~K}$, air velocity $V_{\text {air }}=0.3 \mathrm{~m} / \mathrm{s}$ and the mass fraction of $\mathrm{O}_{2}$ is $23 \%$. The initial droplet temperature, $T_{\mathrm{i}}=300 \mathrm{~K}$, is uniform and low compared to the boiling point. The two droplets are aligned along the free stream direction, and the droplet center spacing is initially $S$. Table 1 shows the numerical simulated cases that we calculated. For all the cases, the ambient conditions are the same, the initial velocities of two droplets are $0.05 \mathrm{~m} / \mathrm{s}$ and the initial droplet center spacing $S$ is $150 \mu \mathrm{m}$.The following assumptions are applied in the forthcoming 
simulations: (1) The buoyancy effects and the thermal radiation effects are neglected.(2) Phase equilibrium is maintained at the gas-liquid interphase.(3) Air is insoluble in the liquid phase.(4) Dufour (energy flux due to mass concentration) and Soret effects (mass diffusion due to temperature) are assumed negligible.(5) The Marangoni effect, that is, interfacial shear stress caused by the variations of surface tension due to temperature is not modeled. The mumerical calculations for an n-octane droplet by $\mathrm{Wu}$ and Sirignano ${ }^{[11]}$ showed that the effects of surface tension on the droplet surface temperature and droplet mass transfer rate are small, especially in the later stage of droplet burning and even there are no effects.

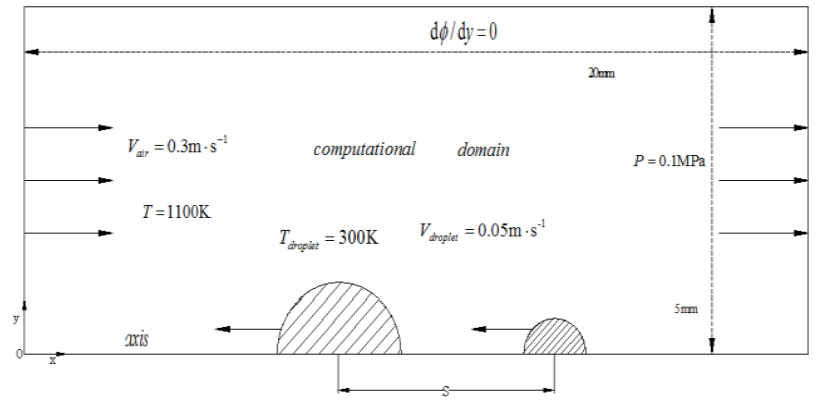

Figure 1. Physical configuration of two different n-decane droplets burning

TABLE 1. Numerical simulated cases for two droplets burning at

different droplet radius ratios with fixed droplet center spacing

\begin{tabular}{|c|c|c|c|}
\hline $\begin{array}{c}\text { Calculation } \\
\text { conditions: }\end{array}$ & $\boldsymbol{R}_{\mathbf{1}}$ (the front \\
$V_{\text {air }}=0.3 \mathrm{~m} \cdot \mathrm{s}^{-1}$ & $\boldsymbol{R}_{\mathbf{2}}$ (the back \\
droplet) & $\begin{array}{c}\text { droplet) } \\
T_{\text {air }}=1100 \mathrm{~K}\end{array}$ & $\begin{array}{c}\text { Droplet } \\
\text { radus } \\
\text { ratio } \phi \\
T_{\text {droplet }}=300 \mathrm{~K}\end{array}$ & $\begin{array}{c}\mu \mathrm{m} \\
\left(\phi=\boldsymbol{R}_{\mathbf{1}} /\right. \\
\left.\boldsymbol{R}_{\mathbf{2}}\right)\end{array}$ \\
\begin{tabular}{c}
$\boldsymbol{P}=0.1 \mathrm{MPa}$ \\
The initial \\
droplet center \\
\cline { 2 - 4 } spacing
\end{tabular} & 42.5 & 50 & 0.85 \\
\cline { 2 - 4 }$S=150 \mu \mathrm{m}$ & 45 & 50 & 0.90 \\
\cline { 2 - 4 } The mass & 47 & 50 & 0.94 \\
\cline { 2 - 4 } fraction of $\mathrm{O}_{2}$ & 49 & 50 & 0.98 \\
\cline { 2 - 4 } is $23 \%$ & 50 & 49 & 1.02 \\
\cline { 2 - 4 } & 50 & 47 & 1.06 \\
\cline { 2 - 4 } & 50 & 42.5 & 1.18 \\
\hline
\end{tabular}

III. MATHEMATICAL MODEL

\section{A. Mass transfer model}

The VOF mathematical formulation for two-phase flow is a single-fluid model and only comprises a set of governing equations for both phases. The fields for all variables and properties are shared by the phases and represent volume-averaged values. Mass transfer between gas and liquid is the key to this simulation. The total mass transfer per volume from the liquid to gas is ${ }^{[12]}$

$$
m_{i, v}=\frac{-M_{i} D_{i, m} d c_{i} / d l}{1-x_{i}} A_{s} / V_{c}
$$

This mass transfer equation is deduced from the classical diffusion equation, where $x_{\mathrm{i}}$ is the molar fraction of species $i$ and $A_{\mathrm{S}}$ is the mass transfer area between phases in cell on gas-liquid interface. Concentration gradients of mass transfer between the liquid phase and gas phase $d c_{\mathrm{i}} /$ $d l$ are calculated by following formula

$$
-d c_{i} / d l=\left(c_{i, s}-c_{i, g}\right) / L
$$

where, $c_{\mathrm{i}, \mathrm{s}}$ is the molar concentration of species $i$ at liquid surface, and $c_{\mathrm{i}, \mathrm{g}}$ represents the concentration of species $i$ in the gas mixture at the same cell, and $L$ stands the unit cell length in the droplet motion area.

The core of the VOF method is the volume fraction. In order to increase the stabilities of numerical calculation(the slow transition of density), the gas-liquid interface will be smeared over at least one grid cell, that is, there is no obvious gas-liquid interface, but a transition area. However, it significantly enhances the heat and mass transfer between the phases, resulting in a faster evaporation of the droplet than that in reality. It is assumed that the convective contribution from every cell is simply proportional to the gas volume fraction in the cell, which quickly decreases from the cell near liquid phase to that near the gas phase in the transition zone. Finally, mass transfer rate is written as

$$
m_{i, v}=K \alpha_{l} \alpha_{g} \alpha_{g} M_{i} D_{i, m}\left(c_{i, s}-c_{i, g}\right) /\left[\left(1-\alpha_{g} x_{i}\right) \cdot V_{c}\right]
$$

When the liquid volume fraction in the transition area is less than $1 \%$, the temperature may reach its boiling temperature. In this case, we used a boiling model as that in [13].

\section{B. Chemical reaction model}

For the auto-ignited combustion of decane in air, the assumed irreversible one-step reaction written in general form is

$$
a \mathrm{C}_{10} \mathrm{H}_{22}+b \mathrm{O}_{2}=\mathrm{cH}_{2} \mathrm{O}+d \mathrm{CO}_{2}
$$

The Arrhenius expressions based on the laminar flow finite-rate model is

$$
\begin{gathered}
R_{C_{10} H_{22}}=-M_{C_{10} H_{22}} a A e^{-E_{a} / R T}\left(c_{C_{10} H_{22}}\right)^{\eta_{C_{10} H_{22}}\left(c_{O_{2}}\right)^{\eta_{O_{2}}}} \\
\text { where } \quad \eta_{C_{10} H_{22}}=0.25 \quad, \quad \eta_{O_{2}}=1.5
\end{gathered}
$$

$E_{a}=1.256 \times 108 \mathrm{~J}_{\square \mathrm{kmol}^{-1}}$ and $A=2.562 \times 10^{9}$ for decane ${ }^{[14]}$.

The properties of gas phase are temperature dependent and species dependent. In addition to surface tension, the properties of liquid phase are temperature dependent.

\section{MODELING PROCESS}

The governing equations are solved numerically on two-dimensional structured four-square grid. To model the process of heat and mass transfer in the gas-liquid interface accurately, the grids are refined in the domain of $1 \mathrm{~mm}$ long and $0.07 \mathrm{~mm}$ high along the flow direction. The unit cell length is $1.25 \mu \mathrm{m}$ in this domain. The ratios of the unit cell length and the droplet initial diameters are less than the value of the grid independent solution in [15].Therefore, the effect of grid number on numerical results can be ignored. The time step is $\Delta \mathrm{t}=0.5 \mu \mathrm{s}$ which is less than the droplet characteristic time $t_{c}\left(t_{c}=L / v_{1} \approx 1.25(\mu \mathrm{m}) / 0.05(\mathrm{~m} / \mathrm{s})=25 \mu \mathrm{s}, v_{1}\right.$ is droplet 
velocity). We take $10^{-3}$ as the convergence criterion for the residual for the continuity equation and the species equations, and $10^{-6}$ for the energy equation, respectively. The pressure-implicit with splitting of operator (PISO) method is used for pressure-velocity coupling. PRESTO! is used to calculate the pressure. The geometric reconstruction approach is used to solve the volume fraction equations of species.

\section{RESULTS AND DISCUSSIONS}

\section{A. Model validation}

In order to guarantee the reliability of the numerical simulation results, the combustion process of the droplet at the ambient temperature of $300 \mathrm{~K}$ with different air stream velocities is numerically simulated firstly. The burning rate constants of the decane droplet at different air stream velocities are measured in the literature $[2,16]$. It can be seen from Figure 2 that the variation trend of the burning rate constants in numerical simulation is consistent with that in the experiment. Also, the differences between simulated results and the experimental results are small. The ignition time accounted for the proportion of the droplet entire burning time is greater than that in the experiment. The highest temperature at some region is larger than $2000 \mathrm{~K}$ in the numerical simulation, so the ignition of droplet actually happens at the ambient temperature greater than $1500 \mathrm{~K}$ in the numerical simulation. In a word, the numerical results accord well with the experimental results This verify the established mass transfer model and boiling model correct and feasible, so the combustion of two droplets at different radius ratios is studied with established models.

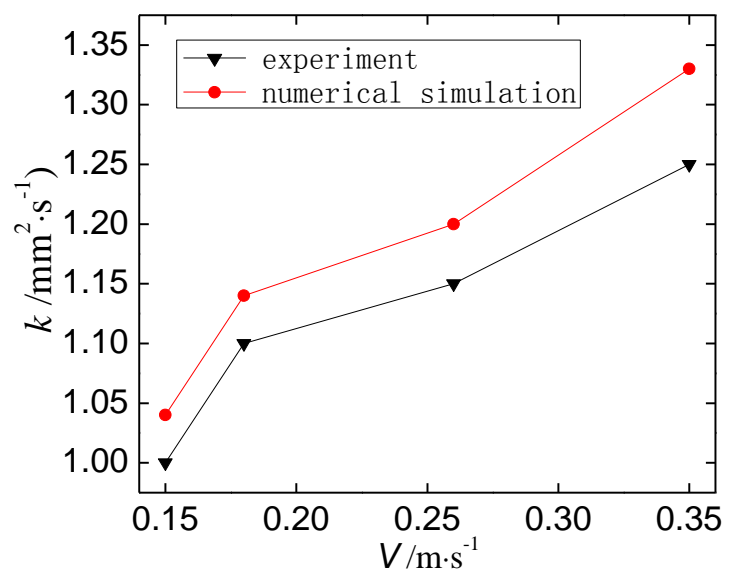

Figure 2. The comparisons of burning rate constants amongst the experimental results in the literature $[2,16]$ and the results in our numerical simulation at different air stream velocities

\section{B. Effect of droplet initial diameter on the burning rate of a single droplet}

The variation of diameter-squared of the single droplet at various initial droplet radiuses is shown in Figure 3. Figure 3 shows the droplet diameter increases firstly and then decreases in the droplet lifetime. This is due to the change of thermal property of the liquid phase. The expansion rate of droplet is larger than the evaporation rate of droplet surface in the initial stage, so the droplet diameter increases firstly and then decreases. It can be seen from Figure 3 that these curves approximate to linear variation and basically meets $\mathrm{D}^{2}$ law in most of droplet lifetime. When the initial droplet radius increases, the curve of droplet becomes slower. That is, the burning rate of droplet is smaller as the initial droplet radius increases. The reason is the temperature of larger droplet from the room temperature to the saturation temperature increases slower at the same quantity of heat condition. Therefore, the burning rate of the larger droplet is smaller. The lifetime of the droplet increases with the increase of the initial radius of the droplet, as shown in Figure 3. The reason is that the burning rate of droplet decreases as the initial radius of the droplet increases, so the lifetime of the droplet increases correspondingly.

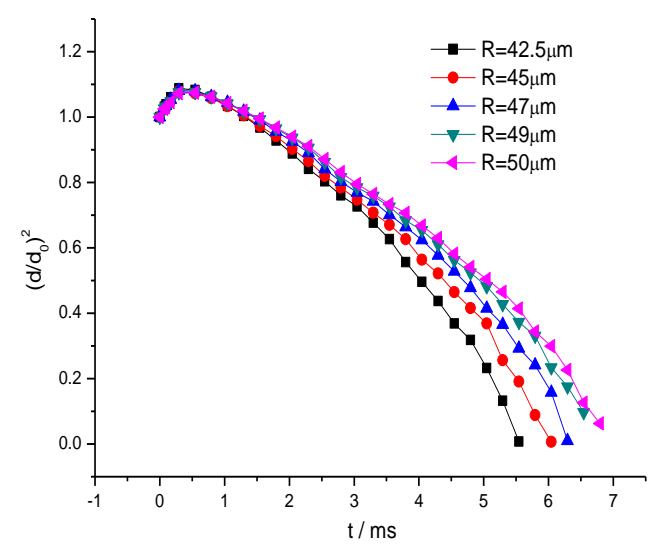

Figure 3. The variation of diameter-squared of the single droplet at various initial droplet radiuses

\section{Effect of droplet radius ratio on the burning rates of two droplets}

The comparisons of the histories of diameter-squared amongst the same initial radius droplet and the corresponding single droplet at different droplet radius ratios are shown in Figure 4 and Figure 5. Figure 4 and Figure 5 show the droplet diameter increases firstly and then decreases. It can be seen from Figure 4 and Figure 5 that these curves approximate to linear variation and basically meets $\mathrm{D}^{2}$ law in most of droplet lifetime. When the burning rates of the front and the back droplet compare with that of the corresponding single droplet, the interaction reduces the burning rate of either droplet under different droplet radius ratios, as shown in Figure 4 and Figure 5. Figure 4 and Figure 5 also show that the burning rate of droplet located at the front is larger than that at the back for the same initial droplet radius under different droplet radius ratios. This is due to the following two reasons: the first one is that the low-temperature vapor of the front droplet is blew to the downstream directly and the temperature around the back droplet decreases; the second one is that the existence of the front droplet directly affects the flow field distribution of the back droplet and reduces the airflow velocity and convection. 


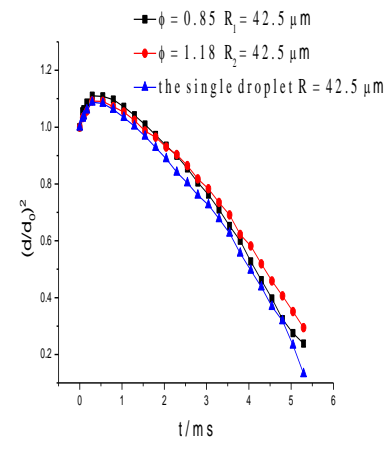

(a) $\mathrm{R}=42.5 \mu \mathrm{m}$

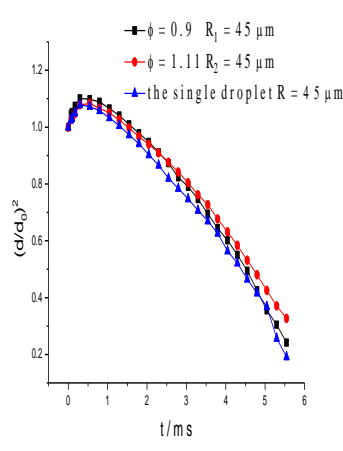

(b) $\mathrm{R}=45 \mu \mathrm{m}$

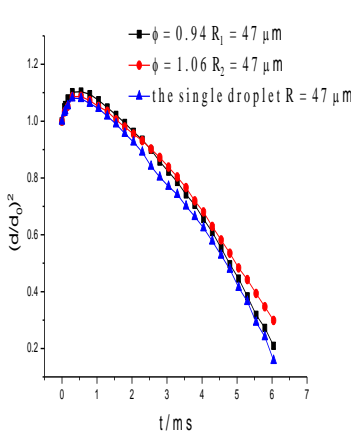

(c) $\mathrm{R}=47 \mu \mathrm{m}$

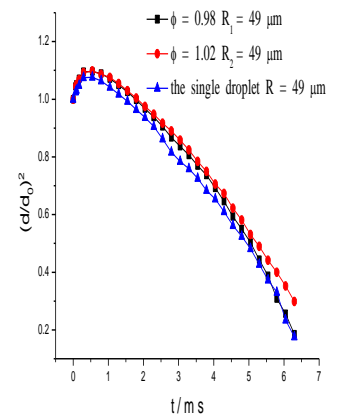

(d) $\mathrm{R}=49 \mu \mathrm{m}$

Figure 4. The comparisons of the evolution of diameter-squared amongst the same initial radius droplet and the corresponding single droplet at different droplet radius ratios
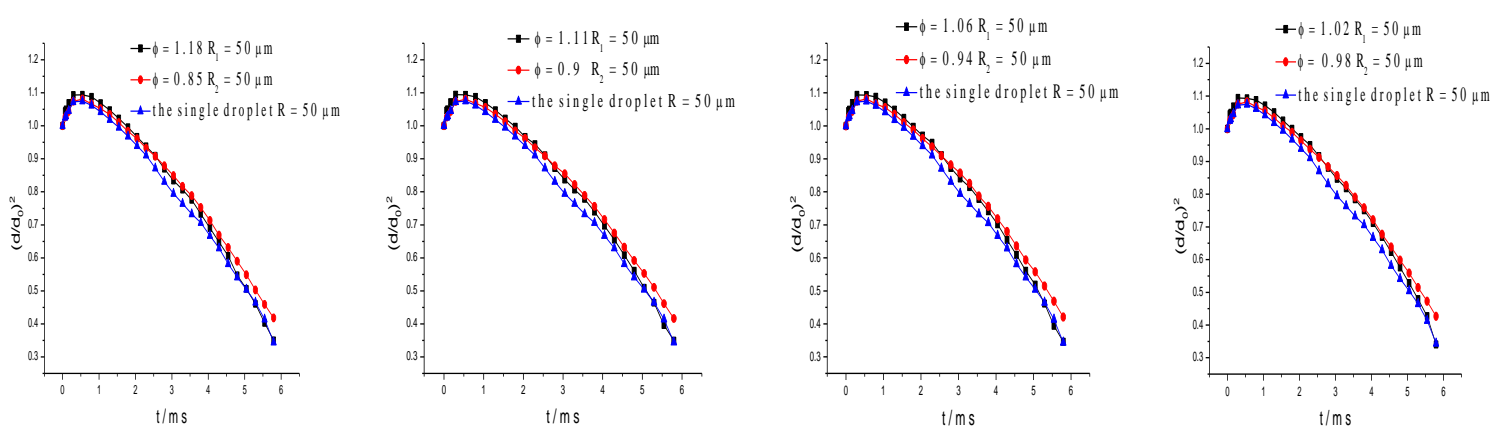

Figure 5. The comparisons of the histories of diameter-squared amongst the same initial radius droplet and the corresponding single droplet at different droplet radius ratios

\section{CONCLUSIONS}

The volume of fluid (VOF) formulation is applied to model the combustion process of two decane droplets with different diameters in a hot convective flow and the effects of the droplet ratios on burning rates of two droplets at fixed droplet center spacing are analyzed. The droplet deformation is considered in the VOF method compared with the traditional point source method, which is closer to the actual combustion. From the above analysis, the conclusions of this study are as follows:

1. When the initial diameter of the single droplet increases, the burning rate of the single droplet decreases and the lifetime of the single droplet increases correspondingly. 2. When the burning rates of the front and the back droplet are compared with that of the corresponding single droplet, the interaction reduces the burning rate of either droplet under different droplet radius ratios.

3. For the same initial droplet radius, the burning rate of droplet located at the front is larger than that at the back under different droplet radius ratios.

\section{REFERENCES}

[1] Sirignano W A, Fluid Dynamics and Transport of Droplets and Sprays, New York: Cambridge University Press, 1999.

[2] Xu G W, Ikegami M, Honma S, et al., "Inverse Influence of Initial Diameter on Droplet Burning Rate in Cold and Hot Ambiences: a Thermal Action of Flame in Balance with Heat Loss," International Journal of Heat and Mass Transfer, vol. 46, Mar. 2003, pp. 1155-1169, doi:10.1016/S0017-9310(02)00397-6.

[3] J.H. Bae 1, C.T. Avedisian, "Experimental study of the effect of helium/nitrogen concentration and initial droplet diameter on nonane droplet combustion with minimal convection,"
Proceedings of the Combustion Institute, vol. 31, Jan. 2007, pp. 2157-2164, doi:10.1016/j.proci.2006.08.042.

[4] D.P. Mishra, Advitya Patyal, "Effects of initial droplet diameter and pressure on burning of ATF gel propellant droplets," Fuel, vol. 95, May 2012, pp. 226-233, doi:10.1016/j.fuel.2011.09.041.

[5] Shinji Nakaya, Kotaro Fujishima, Mitsuhiro Tsue, Michikata Kono, Daisuke Segawa, "Effects of droplet diameter on instantaneous burning rate of isolated fuel droplets in argon-rich or carbon dioxide-rich ambiences under microgravity," Proceedings of the Combustion Institute, vol. 34, 2013, pp. 1601-1608, doi:10.1016/j.proci.2012.05.086.

[6] T.A. Brzustowski, E.M. Twardus, S.Wojcicki, A.Sobiesiak, "Interaction of Two Burning Fuel Droplets of Arbitrary Size," AIAA Journal, vol. 17, 1979, pp. 1234-1242.

[7] A.Umemura, S.Ogawa, N.Oshima, "Analysis of the interaction between two burning fuel drops with different sizes," Combustion and Flame, vol. 43, 1981, pp. 111-119, doi:10.1016/00102180(81)90010-9.

[8] Raju M.S., Sirignano W.A., "Interaction between two vaporizing droplets in an intermediate Reynolds number flow," Physics of Fluids, vol. 2, 1990, pp. 1780-1796.

[9] RaghavanV.,BabuV.,SundararajanT., "Investigation of interaction between methanol fed tandem porous spheres burning in a mixed convective environment," COMBUSTION THEORY AND MODELLING, vol. 13, 2009, pp. 461-485, doi: $10.1080 / 13647830902822198$.

[10] Chiang C H, Sirignano W A., "Interacting, Convecting, Vaporizing Fuel Droplets with Variable Properties," International Journal of Heat and Mass Transfer, vol. 36, Mar. 1993, pp. 875886, doi:10.1016/S0017-9310(05)80271-6.

[11] Guang W, Sirigano W A., "Transient burning of a convective fuel droplet," Combustion and Flame, vol. 157, May 2010, pp. 970 981, doi:10.1016/j.combustflame.2009.11.019. 
[12] ZHANG Xiaobin, XIANG Shijun, CAO Xiaoli, ZHANG Xuejun, "Numerical Simulation for Combustion of Droplet with Volume of Fluid Formulation," CIESC Journal, vol. 62, Mar. 2011, pp. 692698, doi:0438-1157(2011)03-0692-07.

[13] Zhang Xiaobin , Zhang Wei, Zhang Xuejun, "Modeling dropletvaporization and combustion with the volume of fluid method at a small Reynolds number," Journal of Zhejiang University- SCIENCE A (Applied Physics \& Engineering), vol. 13, 2012, pp. 361-374, doi:10.1631/jzus.A1100338.

[14] Westbrook C K, Dryer F L., "Simplified reaction mechanisms for the oxidation of hydrocarbon fuels in flames," Combustion Science and Technology, vol. 27, 1981, pp. 31 43,doi:10.1080/00102208108946970.

[15] Chiang C H, Raju M S, Sirignano W A, "Numerical analysis of convecting, vaporizing fuel droplet with variable properties," International Journal of Heat and Mass Transfer, vol. 35, May 1992, pp. 1307 -1324, doi:10.1016/0017-9310(92)90186-V.

[16] Xu G W, Ikegami M, Honma S, et al., "Interactive Influence of Convective Flow and Initia1 Droplet Diameter on Isolated Droplet Burning Rate," International Journal of Heat and Mass Transfer. vol. 47, Apr. 2004, pp. 2029-2035, doi: 10.1016/j.ijheatmasstransfer.2003.09.035 